\title{
A photonic beamformer based on complex-valued filtering of wavelength-division multiplexed signals
}

Rui Oliveira, Rogério Nogueira, Miguel Drummond

Rui Oliveira, Rogério N. Nogueira, Miguel V. Drummond, "A photonic beamformer based on complex-valued filtering of wavelength-division multiplexed signals," Proc. SPIE 11852, International Conference on Space Optics - ICSO 2020, 118523 S (11 June 2021); doi: 10.1117/12.2599621

SPIE Event: International Conference on Space Optics - ICSO 2021, 2021, Online Only 


\section{International Conference on Space Optics-ICSO 2020}

Virtual Conference

30 March-2 April 2021

Edited by Bruno Cugny, Zoran Sodnik, and Nikos Karafolas
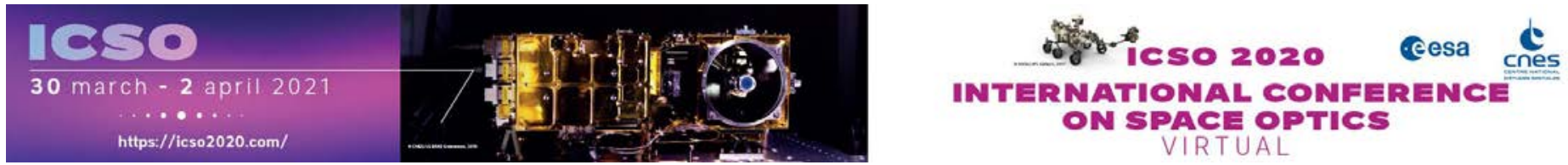

\section{A photonic beamformer based on complex-valued filtering of wavelength-division multiplexed signals}

\section{Cesa isoporeasedngs denes}




\title{
A photonic beamformer based on complex-valued filtering of wavelength-division multiplexed signals
}

\author{
Rui Oliveira, Rogério N. Nogueira, and Miguel V. Drummond \\ Instituto de Telecomunicações and Universidade de Aveiro, Aveiro, Portugal \\ \{ruilvo, rnogueira, mvd\}@av.it.pt
}

\begin{abstract}
In this work, we present a novel microwave photonic signal processor tailored for beamforming large-scale phased array antennas. Theoretical, numerical, and experimental validation is reported for a phased array antenna receiver. Results demonstrate the possibility of separating multiple spatially multiplexed beams using this system. At the core of the design is the use of wavelength division multiplexing and a programable photonic processor, capable of producing dynamic, complex-valued, and arbitrary optical filtering. The scalability and applicability in space applications is discussed.
\end{abstract}

Keywords: Radio frequency, photonics, beamforming, satellite communications

\section{INTRODUCTION}

Communications satellites are in a singular position to provide ubiquitous digital access, as land-based systems cannot viably reach everywhere. To achieve this goal, the economics of satellite access must become comparable with land-based systems.

To make satellite communications cost-effective, the coverage and throughput of each individual system ought to be maximized. Traditionally, this has been done by packing ever more beams into a satellite payload. ${ }^{1}$ This solution is at the limits of its scalability. Furthermore, the decades-long deployments for communication satellites make static solutions a commercial risk, as these are not able keep up with the fast-changing changing requirements on the ground. Thus, satellites must provide flexible coverage.

At the centre of flexible coverage is dynamic beamforming. However, to the best of our knowledge, beamformed antennas that provide satellite-friendly size, weight, and power consumption (SWaP) metrics using traditional electronics solutions have yet to reach the market. In this context, photonic-aided payloads have been identified as a promising technology to achieve flexible coverage with suitable SWaP metrics. ${ }^{2,3}$

In this paper, we present a novel microwave photonic signal processor (MPSP), and its applications for beamforming of microwave signals. The system is based on arbitrary-shaped and complex valued filtering of wavelength-division multiplexing (WDM) signals. Both an analytical description and numeric simulations are presented, as well as a proof-of-concept experiment, demonstrating receive-end beamforming of a single beam, and the separation of multiple beams. The operation principle of the proposed beamformer is validated by numerical and experimental results, as the beamformed beams are correctly recovered. Finally, we discuss advantages and challenges for the application of the system proposed in the context of satellite communications.

\section{ARCHITECTURE OVERVIEW}

To understand the working principle behind the proposed MPSP, let us look into its use in a receiving beamformer for a phased array antenna (PAA). Such application is depicted in figure 1.

The signals produced by the antenna elements (AEs) from a PAA are modulated into the optical domain using optical single-sideband with carrier $(\mathrm{SSB}+\mathrm{C})$ modulation, performed by Mach-Zehnder modulators (MZMs). The laser sources feeding each modulator are tuned to different wavelengths, so that the modulated signals do not occupy the same spectral region. These optical signals are then multiplexed together using an optical multiplexer. At this point, we have a wavelength-division multiplexing (WDM) signal, containing a series of optical carriers and RF sidebands, corresponding to each AE. 


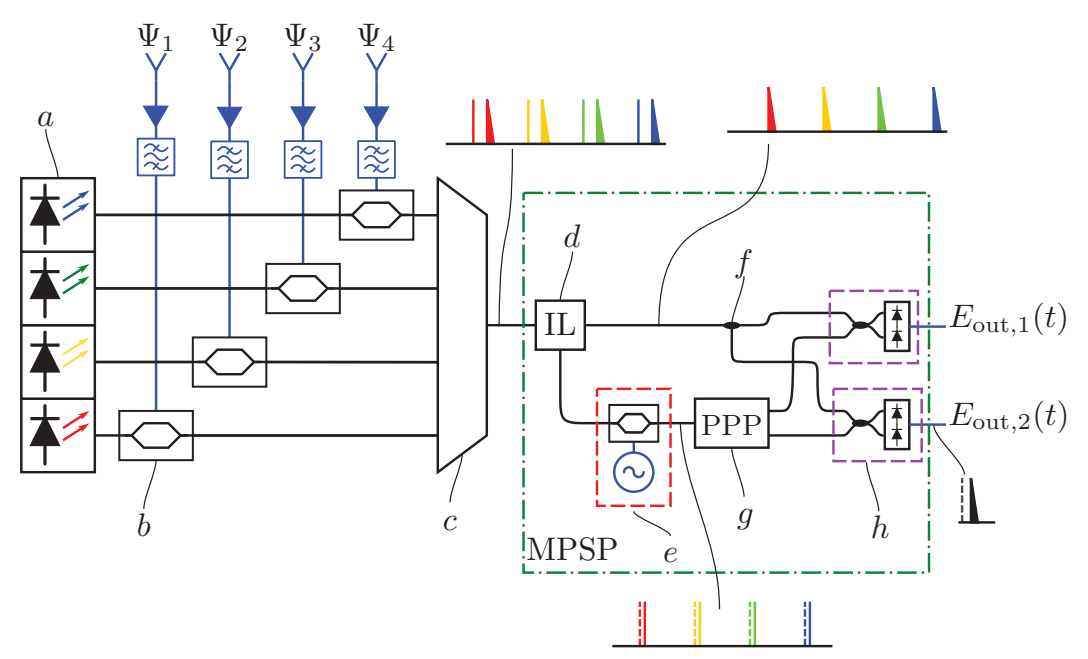

Figure 1. Proposed receive-side beamformer and MPSP for four antenna elements and two output beams. The MPSP is comprised of the elements inscribed in the dot-dash rectangle. Illustrations of the optical spectrum at various points, and the RF spectrum at the output are displayed. $\Psi_{i}$ denotes the signal shinning on the i-th antenna element, and $E_{\text {out }, k}$ denotes the k-th beamformed output beam. (a) lasers to be modulated by each modulator; (b) single-sideband w/ carrier optical modulators; (c) optical multiplexer; (d) optical interleaver; (e) optical local oscillator generation; (f) $3 \mathrm{~dB}$ splitter; (g) programmable photonic processor; (h) coherent optical detector.

\subsection{The microwave photonics signal processor}

This WDM signal generated in the modulation step is then fed to what we hereby define as the microwave photonic signal processor (MPSP). As the name implies, this device has been designed specifically for dealing with microwave-range RF signals, by processing them in the optical domain. Its architecture is closely coupled with the SSB+C modulation (plus multiplexing) previously presented. Its working principle relies in the association between complex-valued filtering in the optical domain and its correspondence in the RF domain. In fact, with this kind of setup, changing the amplitude and/or phase of the optical signal directly translates in the same amplitude and/or phase modulation in the RF signal. ${ }^{4}$ This property is key to understand the beamforming ability of the MPSP.

The first task the MPSP has to perform is to split the optical carriers from the RF sidebands into two separate WDM signals. For this, an optical interleaver is used. These two signals constitute the arms of what is essentially a Mach-Zehnder interferometer.

The WDM signal containing exclusively RF sideband is not further processed, and routed directly to one of the ports of a coherent detector. Rather, all the effects imposed on the output happen by processing only the optical carriers. For scenarios where multiple simultaneous outputs are desired, as is the case in simultaneous multibeam beamforming, this signal can be split with an optical splitter and fed to multiple coherent detectors.

Focus then turns to the other WDM signal, containing the optical carriers, preserved in the modulation step. A local oscillator feeding an additional single-sideband MZM is used to frequency-shift the optical carriers. This effectively generates an intermediate frequency (IF) stage, so that the output signals are up or downconverted.

The next stage in this arm presents the programmable photonic processor (PPP). The PPP is defined as a device capable of arbitrary, complex-valued, and dynamic filtering of optical signals. Devices based on LCoS technology capable of this feature have been presented. ${ }^{5,6}$ This is where the key operation in the system happens. Conceptually, each optical carrier is multiplied by a complex-valued factor, and that results in the same factor applied to the respective RF output. In the particular case of beamforming, the optical carriers serve as the steering mechanism of the beamformer. Their relative amplitude and phase relations result in the same effect as any beamsteering performed, for example, in the pure analogue domain. Effectively, this is the stage where the complex-valued weighting of the AEs of the PAA is done. Once again, if multiple outputs are desired, a 
PPP with one-to-many routing capabilities can be used, and the different copies of the optical carriers processed independently.

The last stage is coherent detection. Self-heterodyne and coherent detection has already been extensively covered elsewhere. ${ }^{4,7}$ However, in the particular case of beamforming a PAA, the situation gets nuanced due to the WDM nature of the optical signals involved. In theory, this would result in a multitude of components being generated at the output of the detector, resulting from the product between every combination of optical carriers and RF sidebands. In practice, most of these component are out of the detection range of typical detectors. In fact, given enough separation between the optical carriers, only the product between a given optical carrier and its respective RF sideband contributes to the output RF signal. These aforementioned products between the optical carriers and their respective RF sidebands produce superimposed outputs. Once again, this is analogous to using RF combiners in a traditional beamforming scenario. The combined output signals, weighted by the applied complex-valued factors result in the desired beamforming operation.

\subsection{Architectural considerations}

The proposed beamformer and MPSP present a combination of features that make it an interesting option for space-related applications, both spaceborne and in the ground.

WDM is a mature technology, which is a key facilitator in the introduction of photonics into an otherwise conservative industry. And, more important, its biggest practical advantage is the minimization of the number of physical channels. This is key in SWaP-constrained systems, as is the case of satellite payloads. Effectively, the proposed system replaces multiple RF transport media (coaxial cabling, rigid waveguides, etc.) with a single optical fibre. The use of LCoS-based phase shifting is also a key enabling technology: a full-HD LCoS panel essentially has two million phase shifters which provides unmatched processing density. Finally, the reliance of self-heterodyne detection is supported by previous successes. ${ }^{1,4,7-9}$ In particular, the integrated frequency conversion capability, alongside phase noise tolerance, make it a first-class optical detection technique.

In the case of communications satellites, this system also has the added benefit of allowing the beamformer to be arbitrarily positioned inside the payload, increasing design flexibility. In fact, the feasibility of spacequalification for LCoS technology is already a subject of study. ${ }^{10,11}$

For ground-based operations, the system presents two main lines of potential industrial application. On one hand, there is potential to be an enabler for SWaP-optimized flat-panel antennas for base-stations. And, parallelly, there is potential for enabling beamforming in cellular base stations, while at the same time allowing the optical modulation and the MPSP to be physically distant, taking advantage of radio-over-fibre techniques.

\section{ANALYTICAL MODEL}

In this section we present detailed mathematical motivation behind the working principles of the MPSP in the context of a receiver-end beamformer.

A radio signal shinning over a PAA, with carrier frequency $\omega_{\mathrm{RF}}$ can generally be described as:

$$
\Psi(t)=A(t) \exp \left(j\left(\omega_{\mathrm{RF}}(t) t\right)\right),
$$

where $A(t)$ and $\omega_{\mathrm{RF}}(t)$ describe the signal's instant amplitude and frequency respectively, and $j$ is the imaginary unit.

Without loss of generality, this mathematical analysis assumes a linear PAA. Assuming the non-trivial case of the beam not shinning directly over a linear PAA, each consecutive antenna element (AE) will receive a delayed version of the incoming signal. The delay between consecutive AEs is given by: ${ }^{12}$

$$
\delta t=d / c \times \sin (\alpha),
$$

where $d$ is the distance between AEs, $c$ is the speed of light, and $\alpha$ is the angle the beam makes with the normal of the PAA. The signals at each $\mathrm{AE}, \Psi_{i}(t)$, are defined as:

$$
\Psi_{i}(t)=A(t-i \delta t) \exp \left(j \omega_{\mathrm{RF}}(t-i \delta t) t\right) .
$$


Here, two approximations shall be made. First, a zeroth-order approximation for the amplitude component:

$$
A(t-i \delta t) \approx A(t) \forall i,
$$

and a first order approximation in the phase component:

$$
\exp \left(j \omega_{\mathrm{RF}}(t-i \delta t) t\right) \approx \exp \left(j\left(\omega_{\mathrm{RF}}(t) t-i \delta \theta\right)\right) \forall i,
$$

with $\delta \theta=\omega_{\mathrm{RF}} \delta t$. This finally leaves us with the approximated version of $\Psi_{i}(t)$ :

$$
\Psi_{i}(t) \approx A(t) \exp \left(j\left(\omega_{\mathrm{RF}}(t) t-i \delta \theta\right)\right) .
$$

As previously discussed, the signals from each $\mathrm{AE}$ are modulated into the optical domain at different frequencies, $\omega_{\mathrm{o}, i}$, and multiplexed together using a SSB $+\mathrm{C}$ modulation scheme, and an optical multiplexer. At the output of the optical multiplexer we can describe the signal as:

$$
M(t)=\sum_{i=0}^{N-1} \frac{\sqrt{P_{i}}}{2} \exp \left(j\left(\omega_{\mathrm{o}, i} t+\Phi_{\mathrm{o}, i}(t)\right)\right) \cdot\left[1+\Psi_{i}(t)\right],
$$

where $N$ is the number of AEs, $P_{i}$ in the i-th laser power, and $\Phi_{\mathrm{o}, i}(t)$ accounts for its phase noise, or other phase instabilities in the optical signal

The next optical element in the system is an optical optical interleaver (IL). This component is responsible for separating the optical carriers and the modulated RF sidebands into different output ports. At the output of the IL, there is one signal comprising only of RF sidebands, and another comprising only of optical carriers. We define these signals as $L_{1}$ and $L_{2}$ respectively:

$$
\begin{aligned}
& L_{1}(t)=\sum_{i=0}^{N-1} \Gamma_{i}(t) \Psi_{i}(t), \\
& L_{2}(t)=\sum_{i=0}^{N-1} \Gamma_{i}(t),
\end{aligned}
$$

where $\Gamma_{i}(t)=\left(\sqrt{P_{i}} / 2\right) \exp \left(j\left(\omega_{\mathrm{o}, i} t+\Phi_{i}(t)\right)\right)$.

The signal comprising only optical carriers is routed into an additional MZM. This modulator, connected to a local oscillator with frequency $\omega_{\mathrm{LO}}$, frequency-shifts the optical carriers, causing frequency conversion at the output. At the output of this stage, the optical signal is given by:

$$
L_{2, \mathrm{OLO}}(t)=\sum_{i=0}^{N-1} \Gamma_{i}(t) \exp \left(j\left(\omega_{\mathrm{LO}} t+\Phi_{\mathrm{LO}}\right)\right),
$$

where $\Phi_{\mathrm{LO}}$ is the LO's phase. Again, for convenience's sake, we define $\Upsilon(t)=\exp \left(j\left(\omega_{\mathrm{LO}} t+\Phi_{\mathrm{LO}}\right)\right)$.

The next step is to apply complex-valued filtering to the aforementioned signal. Considering a single beam scenario, the output of the PPP is given by:

$$
L_{2, \operatorname{PPP}}(t)=\sum_{i=0}^{N-1} \Gamma_{i}(t) \Upsilon(t) \exp \left(j \rho_{i}\right),
$$

where $\rho_{i}$ is the phase applied by the PPP to the i-th optical carrier. In our scenario of interest $\rho_{i}=i \delta \theta$.

Finally, signals $L_{1}(t)$ and $L_{2, \mathrm{PPP}}(t)$ are fed as inputs to a coherent, differential optical receiver. Internally, a coherent receiver comprises an two-by-two optical combiner, followed by a differential pair of photodiodes. For the two-by-two optical combiner, its response is given by: ${ }^{13}$

$$
\left[\begin{array}{l}
S_{1} \\
S_{2}
\end{array}\right]=\left[\begin{array}{cc}
\sqrt{1-\epsilon} & j \sqrt{\epsilon} \\
j \sqrt{\epsilon} & \sqrt{1-\epsilon}
\end{array}\right]\left[\begin{array}{c}
L_{1}(t) \\
L_{2, \mathrm{PPP}}(t)
\end{array}\right]
$$


where $\epsilon$ is the combiner's coupling coefficient. Differential detection can be mathematically described as:

$$
E_{\text {out }}(t)=\frac{\mathcal{R}}{Z}\left(\left|S_{1}\right|^{2}-\left|S_{2}\right|^{2}\right)
$$

where $\mathcal{R}$ is the responsivity of the detector and $Z$ its output impedance.

We now work backwards, substituting the defined expressions. First, we use (12) and (13):

$$
E_{\text {out }}(t)=\frac{\mathcal{R}}{Z}\left[\left|\sqrt{1-\epsilon} L_{1}(t)+j \sqrt{\epsilon} L_{2, \mathrm{PPP}}(t)\right|^{2}-\left|j \sqrt{\epsilon} L_{1}(t)+\sqrt{1-\epsilon} L_{2, \mathrm{PPP}}(t)\right|^{2}\right] .
$$

Then, we expand the terms inside the square brackets. Remember that $|x|^{2}=x x^{\dagger}$. The expression yields:

$$
\begin{aligned}
E_{\text {out }}(t)=\frac{\mathcal{R}}{Z} & {\left[(1-2 \epsilon) L_{1}(t) L_{1}^{\dagger}(t)+(2 \epsilon-1) L_{2, \mathrm{PPP}}(t) L_{2, \mathrm{PPP}}^{\dagger}(t)\right.} \\
& \left.+\epsilon(\epsilon-1)\left[L_{1}^{\dagger}(t) L_{2, \mathrm{PPP}}(t)+L_{1}(t) L_{2, \mathrm{PPP}}^{\dagger}(t)\right]\right] .
\end{aligned}
$$

Considering a balanced coherent receiver, $\epsilon=0.5$, the previous equation is left with only the last term:

$$
E_{\text {out }}(t)=\frac{-\mathcal{R}}{4 Z}\left[L_{1}^{\dagger}(t) L_{2, \mathrm{PPP}}(t)+L_{1}(t) L_{2, \mathrm{PPP}}^{\dagger}(t)\right]
$$

Now, both $L_{1}(t)$ and $L_{2, \mathrm{PPP}}(t)$ consist of a sum. In the pure mathematical sense, the product of two sums is not the sum of the products with a single index. But, taking in consideration the physics of the system, any products with different index would correspond to the beating between signals from two different optical carriers. If these are apart enough, their product is well above the bandwidth of a typical optical detector, and so can be disregarded. So, in the next step, the put the sum in evidence, with a single index, consciously disregarding cross products. As another point of simplification, we shall assume all lasers have equal power, i.e., $P_{i}=P \forall i$. This results in:

$$
\begin{aligned}
& E_{\text {out }}(t)=\frac{-\mathcal{R} P A(t)}{16 Z} \sum_{i=0}^{N-1} {\left[\exp \left(j\left(\left(\omega_{\mathrm{LO}}-\omega_{\mathrm{RF}}(t)\right) t+i \delta \theta+\rho_{i}+\Phi_{\mathrm{LO}}\right)\right)\right.} \\
&\left.+\exp \left(j\left(\left(\omega_{\mathrm{RF}}(t)-\omega_{\mathrm{LO}}\right) t-i \delta \theta-\rho_{i}-\Phi_{\mathrm{LO}}\right)\right)\right],
\end{aligned}
$$

which is equal to:

$$
E_{\text {out }}(t)=\frac{-\mathcal{R} P A(t)}{8 Z} \sum_{i=0}^{N-1} \cos \left(\left(\omega_{\mathrm{RF}}(t)-\omega_{\mathrm{LO}}\right) t-i \delta \theta-\rho_{i}-\Phi_{\mathrm{LO}}\right) .
$$

The term $\Phi_{\mathrm{o}, i}(t)$ is absent from this result. This is an expected result from self-heterodyne detection, and has been presented as one of its key advantages. ${ }^{4}$ All terms of the sum become equal if $\rho_{i}=-i \delta \theta$, leaving us with the beamformed signal:

$$
E_{\text {out }}(t)=N \frac{-\mathcal{R} P A(t)}{8 Z} \cos \left(\left(\omega_{\mathrm{RF}}(t)-\omega_{\mathrm{LO}}\right) t-\Phi_{\mathrm{LO}}\right)
$$

The factor $N$, put in evidence, indicates linear scaling with the number of elements, as expected for a beamforming scenario. The stray $\Phi_{\mathrm{LO}}$ phase component is not relevant, as it is no different than any other intermediate frequency (IF) stage in a traditional system.

\section{NUMERICAL ASSESSMENT}

To assess the theorized MPSP, computer simulations were first employed. The simulation consists of one or more virtual RF beams shinning over a virtual PAA. Each beam is comprised of a 1 GBd random QPSK series, carried at $12 \mathrm{GHz}$. The output signal has a carrier frequency of $5 \mathrm{GHz}$, i.e, the beamformer also includes an intermediate frequency (IF) stage. The simulation follows closely the system as presented in figure 1. 

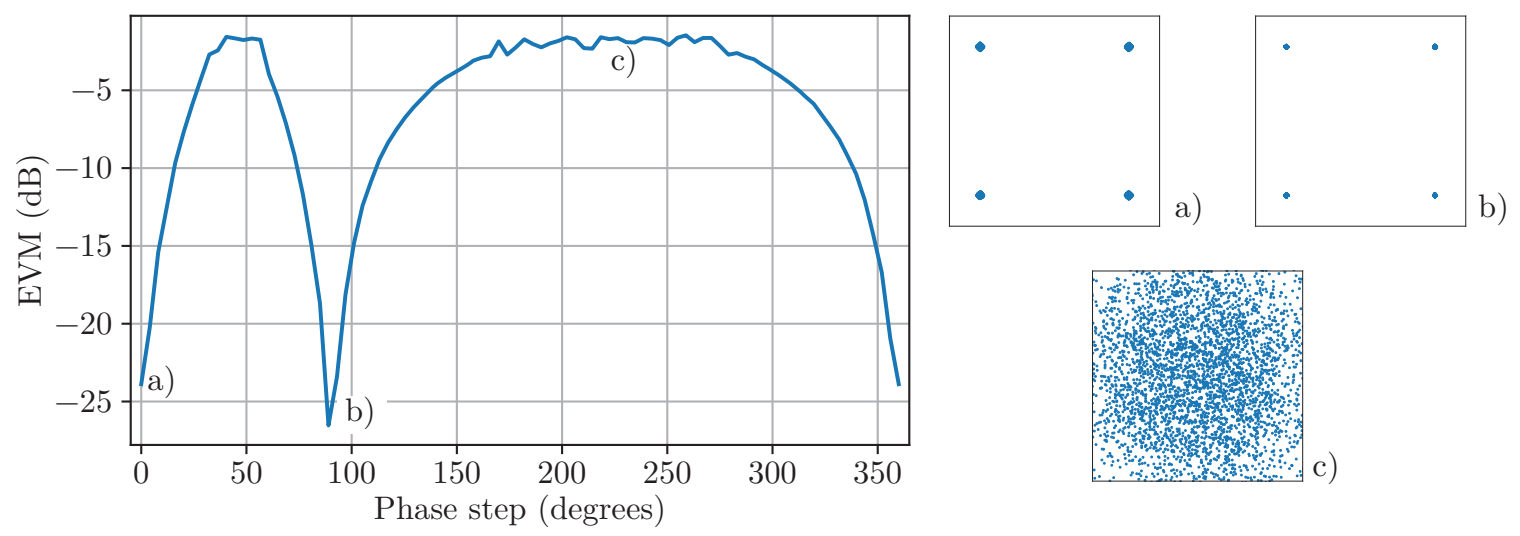

Figure 2. EVM as function of the virtual PAA phase step. a) constellation for the recovered beam at $0^{\circ}$; b) constellation for the recovered beam at $30^{\circ}$; c) beams scrambled when the PPP is not correctly configured.

Without loss of generality, a linear PAA is modelled. First, a WDM signal consisting of SSB signals is generated, closely mimicking the physical implementation. In essence, each SSB signal is comprised on the sum of the delayed versions of the beams at each $\mathrm{AE}$.

The focus of the simulation is the PPP's action. In the simulation, a progressive phase shift step is applied to each frequency-shifted local oscillator (LO). The step of this phase shift between elements is swept, which is analogous to a scanning PAA, like a radar.

After applying the coherent detection step, the output signal is fed to a QPSK demodulator. The final measured metric is the error vector magnitude (EVM). Results for a beamformer with 4 AEs and 2 beams, one shinning at $0^{\circ}$ and another at $30^{\circ}$ are presented in figure 2 . We choose the values of $0^{\circ}$ and $30^{\circ}$ for the beam directions, as these values guarantee that when one of the beams is correctly beamformed the other one is on a null of the radiation pattern of the PAA.

We observe clear separation of two beams, in accordance with the theoretical expectation.

\section{PROOF OF CONCEPT EXPERIMENT}

To provide experimental evidence of the feasibility of the proposed MPSP in the context of a receiving-side beamformer, a proof-of-concept experiment was devised. The parameters used in numerical assessment were considered, such that experimental results can be directly compared with numerical ones, previously presented in figure 2. An illustration of the experimental apparatus is presented in figure 3.

A single laser source is fed into a dual-polarization, IQ, MZM. Each one of the WDM signals as represented by (9), and (10) modulate the laser source in one of the MZM's polarizations each. This signal is fed to a polarization beam splitter (PBS). The separation of the aforementioned signals in the polarization domain makes the PBS essentially function as the optical interleaver in the original design. In essence, one output of the PBS corresponds to the spectrum as seen in the top arm of the interferometer after the optical interleaver, and the other to the spectrum after the optical LO, as depicted in the bottom arm of the same figure. Test data consisted of random QPSK constellations carried $7 \mathrm{GHz}$ above their virtual optical carrier. The optical LOs have a frequency shift of $5 \mathrm{GHz}$, so the system effectively downconverts the beams to $2 \mathrm{GHz}$ at the output of the optical detector. Tests were realized using one or two spatially multiplexed beams. The virtual antenna array contains four elements, limited by the bandwidth of the available arbitrary wave generator (AWG). For the PPP, a wavelength selective switch (WSS) (Finisar 4000S) was used. The electrical output is connected to a real-time sampling oscilloscope. Further processing, including demodulation, is carried offline. 


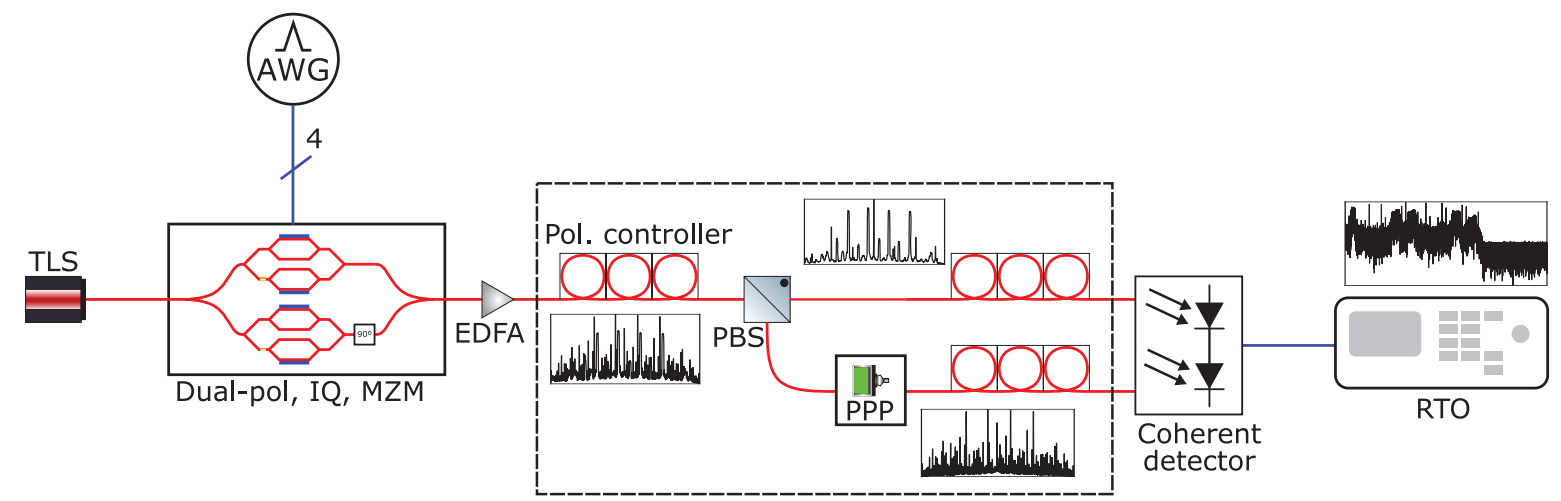

Figure 3. Proof-of-concept experiment for the proposed $\mathrm{Rx}$ beamformer and MPSP. The optical spectrum at different stages is presented. TLS: tuneable laser source; EDFA: erbium-doped fibre amplifier; RTO: real-time oscilloscope.
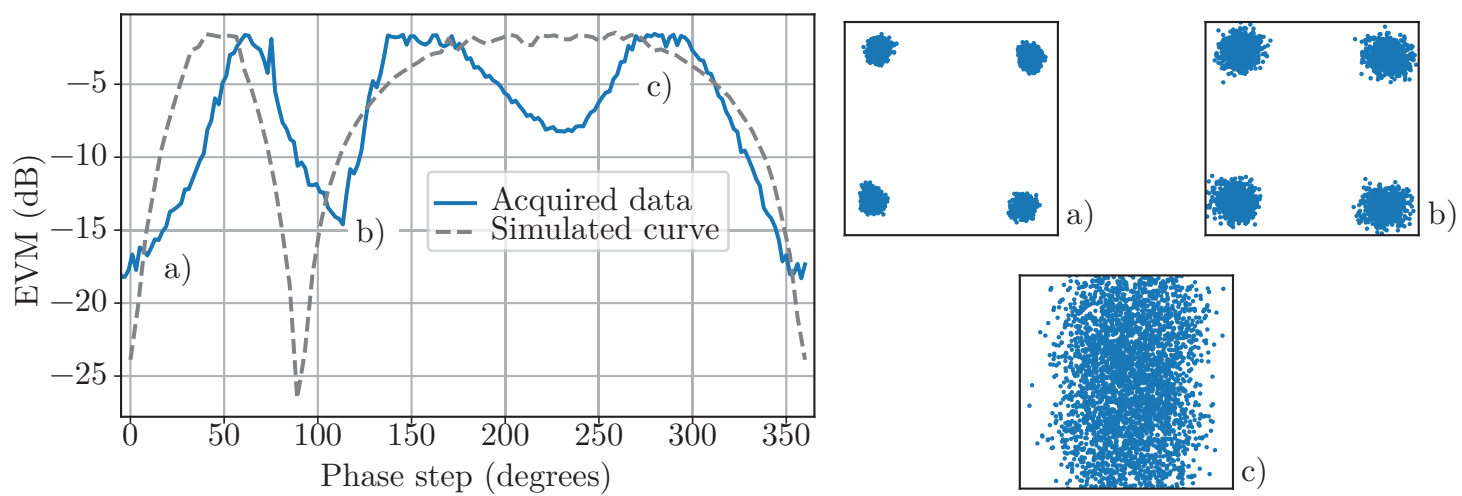

Figure 4. EVM as function of phase step applied on the WSS. a) constellation for the recovered beam at $0^{\circ}$; b) constellation for the recovered beam at $30^{\circ}$; c) beams scrambled when the WSS is not correctly configured.

As with the computer simulations, to demonstrate the beamforming effect, a phase-sweeping routine is implemented over the WSS. Results for a beamforming scenario with four AEs and two simultaneous beams are presented in figure 4.

It is important to discuss the differences between the results of the numerical assessment and the experiment data. First, one can observe shallower valleys and sharper rises in EVM. These are due to the limited dynamic range available in the system, both in the optical modulator, and in the real-time oscilloscope. This is a reasonable result, as the numerical simulation was executed under ideal conditions, including a noiseless environment, and double floating point precision, contrasting to the 8-bit AWG and oscilloscope available.

Second, and more surprisingly, one can observe that the experimental curve lags behind the simulated data. We acknowledge this difference, and we believe this happens due to poor calibration of the WSS used, i.e., the phase requested (and plotted), does not correspond to the actual phase applied by the device. It is worth noting that the limitation to four AEs exacerbates the issue. With a large number of AEs, a random error in calibration would get averaged out. At the time of writing, debugging actions over the experiment are still ongoing.

In spite of such differences between numerical and experimental results, the beamforming effect, by beamsteering a virtual PAA is clearly visible and demonstrated. 


\section{CONCLUSIONS}

A novel MPSP and its use in a receiving beamformer application was reported. The integrated system makes use of WDM and a LCoS-based programmable photonic processor to make a SWaP-friendly system, suitable for spaceborne payloads.

The architecture has been analytically demonstrated, as well as validated both numerically and experimentally. Multiple beam separation at microwave frequencies was demonstrated by an analysis of the sweeping behaviour of the beamformer, using EVM as a figure-of-merit.

Even though this system was originally thought for spaceborne antenna systems, we believe the architecture proposed is suited for any kind of large-scale PAAs, be it in space or in ground systems.

\section{ACKNOWLEDGMENTS}

This work has been funded in part by Fundação para a Ciência e a Tecnologia (FCT) with PhD grant with reference SFRH/BD/146916/2019, by the European Regional Development Fund (FEDER), and by the Regional Operational Programme of Centre (CENTRO 2020) of the Portugal 2020 framework through projects ORCIP (CENTRO-01-0145-FEDER-022141) and RETIOT (POCI-01-0145-FEDER-016432).

\section{REFERENCES}

[1] Duarte, V. C., Prata, J. G., Nogueira, R. N., Winzer, G., Zimmermann, L., Walker, R., Clements, S., Filipowicz, M., Napierala, M., Nasilowski, T., Crabb, J., Stampoulidis, L., Anzalchi, J., and Drummond, M. V., "Modular and smooth introduction of photonics in high-throughput communication satellites perspective of project BEACON," in [International Conference on Space Optics - ICSO 2018], Sodnik, Z., Karafolas, N., and Cugny, B., eds., 11180, 2610-2617, International Society for Optics and Photonics, SPIE (2019).

[2] Inigo, P., Vidal, O., Roy, B., Alberty, E., Metzger, N., Galinier, D., Anzalchi, J., Huggins, G., and Stirland, S., "Review of terabit/s satellite, the next generation of HTS systems," in [2014 "th Advanced Satellite Multimedia Systems Conference and the 13th Signal Processing for Space Communications Workshop (ASMS/SPSC)], 318-322 (Sept. 2014). ISSN: 2326-5949.

[3] Sotom, M., Aveline, M., Barbaste, R., Benazet, B., Kernec, A. L., Magnaval, J., and Picq, M., "Flexible photonic payload for broadband telecom satellites: from concepts to system demonstrators," in [International Conference on Space Optics - ICSO 2016], Cugny, B., Karafolas, N., and Sodnik, Z., eds., 10562, 558-566, International Society for Optics and Photonics, SPIE (2017).

[4] Duarte, V. C., Drummond, M. V., and Nogueira, R. N., "Photonic True-Time-Delay Beamformer for a Phased Array Antenna Receiver based on Self-Heterodyne Detection," Journal of Lightwave Technology 34, 5566-5575 (Dec. 2016).

[5] Baxter, G., Frisken, S., Abakoumov, D., Zhou, H., Clarke, I., Bartos, A., and Poole, S., "Highly programmable wavelength selective switch based on liquid crystal on silicon switching elements," in [2006 Optical Fiber Communication Conference and the National Fiber Optic Engineers Conference], 3 pp.(Mar. 2006).

[6] Robertson, B., Yang, H., Redmond, M. M., Collings, N., Moore, J. R., Liu, J., Jeziorska-Chapman, A. M., Pivnenko, M., Lee, S., Wonfor, A., White, I. H., Crossland, W. A., and Chu, D. P., "Demonstration of MultiCasting in a $1 \times 9$ LCOS Wavelength Selective Switch," Journal of Lightwave Technology 32, 402-410 (Feb. 2014).

[7] Drummond, M. V., Duarte, V. C., Albuquerque, A., Nogueira, R. N., Stampoulidis, L., Winzer, G., Zimmermann, L., Clements, S., and Anzalchi, J., "Dimensioning of a multibeam coherent photonic beamformer fed by a phased array antenna," Optics Express 26, 6158-6171 (Mar. 2018).

[8] Duarte, V. C., Prata, J. G., Ribeiro, C. F., Nogueira, R. N., Winzer, G., Zimmermann, L., Walker, R., Clements, S., Filipowicz, M., Napierała, M., Nasiłowski, T., Crabb, J., Kechagias, M., Stampoulidis, L., Anzalchi, J., and Drummond, M. V., "Modular coherent photonic-aided payload receiver for communications satellites," Nature Communications 10 (Apr. 2019). 
[9] Adleman, J. R., Lin, C. L., Jester, S. B., Pascoguin, B. M., Evans, D. C., and Jacobs, E. W., "Photonic RF-IF wideband down conversion using optical injection locking," in [Micro- and Nanotechnology Sensors, Systems, and Applications VII], George, T., Dutta, A. K., and Islam, M. S., eds., 9467, 360-368, International Society for Optics and Photonics, SPIE (2015).

[10] Silva-López, M., Campos-Jara, A., and Álvarez Herrero, A., "Validation of a spatial light modulator for space applications," in [International Conference on Space Optics - ICSO 2018], 11180, 111806R, International Society for Optics and Photonics.

[11] Otón, E., Pérez-Fernández, J., López-Molina, D., Quintana, X., Otón, J. M., and Geday, M. A., "Reliability of Liquid Crystals in Space Photonics," IEEE Photonics Journal 7, 1-9 (Aug. 2015).

[12] Hansen, R. C., [Phased Array Antennas], John Wiley \& Sons, Inc.

[13] Hui, R. and O'Sullivan, M., [Fiber Optic Measurement Techniques], Academic Press (2009). 